\title{
Oscillating friction of nanoscale capillary bridge
}

\author{
Shuai WU ${ }^{1,2}$, Yuqing $\mathbf{H E}^{3,4}$, Quanshui ZHENG ${ }^{1,2,3}$, Ming MA M $^{2,3,4, *}$ \\ ${ }^{1}$ Department of Engineering Mechanics, Tsinghua University, Beijing 100084, China \\ ${ }^{2}$ Center for Nano and Micro Mechanics, Tsinghua University, Beijing 100084, China \\ ${ }^{3}$ State Key Laboratory of Tribology, Tsinghua University, Beijing 100084, China \\ ${ }^{4}$ Department of Mechanical Engineering, Tsinghua University, Beijing 100084, China \\ Received: 06 September 2019 / Revised: 16 November 2019 / Accepted: 10 April 2020 \\ (C) The author(s) 2020 .
}

\begin{abstract}
The presence of a capillary bridge between solid surfaces is ubiquitous under ambient conditions. Usually, it leads to a continuous decrease of friction as a function of bridge height. Here, using molecular dynamics we show that for a capillary bridge with a small radius confined between two hydrophilic elastic solid surfaces, the friction oscillates greatly when decreasing the bridge height. The underlying mechanism is revealed to be a periodic ordered-disordered transition at the liquid-solid interfaces. This transition is caused by the balance between the surface tension of the liquid-vapor interface and the elasticity of the surface. This balance introduces a critical size below which the friction oscillates. Based on the mechanism revealed, a parameter-free analytical model for the oscillating friction was derived and found to be in excellent agreement with the simulation results. Our results describe an interesting frictional phenomenon at the nanoscale, which is most prominent for layered materials.
\end{abstract}

Keywords: capillary bridge; nano-confinement; elasticity; atomically smooth surface

\section{Introduction}

A capillary bridge, or liquid bridge, is always present between two solid surfaces in contact with or near each other under ambient conditions [1, 2]. Its presence can be due to either thermally activated capillary condensation which is in thermodynamic equilibrium with vapor [3-5], or other non-spontaneous ways, e.g., a tree frog climbing trees by excreting mucus to form capillary bridges between its feet and the tree bark. The great significance of these capillary bridges is not only due to their universal presence, but also their key contribution to the forces between surfaces [6]. The effect of capillary bridges on adhesion has been widely studied since the first observation of a capillary bridge [7]. At the nanoscale, their presence causes interesting behavior which is different with that at the microscale. For example, Cheng and
Robbins found that due to molecular layering of the liquid confined in the narrow gap between the spherical tip and substrate, the adhesion force oscillates strongly with respect to the height of the bridge $[8,9]$. Later Valenzuela et al. show that the oscillation can be suppressed by tuning the values of practical parameters, e.g., tip elastic constant and approach speed [10].

Besides its effect on the adhesion force, the capillary bridge also strongly affects friction between two solid surfaces [11, 12]. In micro- or nano-electromechanical systems (M/NEMS), the capillary bridge often prevents devices from functioning under ambient conditions or leads to damage in their fabrication processes due to the large friction it induces [13]. For example, when the height of the capillary bridge is less than twice the typical atomic sizes, e.g., $0.5 \mathrm{~nm}$, it increases the friction greatly as the water confined between

* Corresponding author: Ming MA, E-mail: maming16@tsinghua.edu.cn 
the surfaces becomes ice-like [14-17]. However, under certain circumstances, the capillary bridge may serve as a lubricant, reducing the intrinsically large friction between solid surfaces [18]. Sometimes, the presence of a capillary bridge can even lead to counter-intuitive friction phenomena. For example, at low sliding velocity, the capillary-mediated friction decreases logarithmically with velocity and increases with temperature $[19,20]$. The mechanism for such intriguing phenomena was revealed to be a combination of thermally activated condensation and the rupture process of the capillary bridge [21, 22].

At present, most of the studies to understand the effects of capillary bridges on friction focus on systems with either multiple capillary bridges or an individual macroscopic capillary bridge. There are limited studies at the nanoscale. By using noncontact dynamic force spectroscopy, Lee et al. studied the shear interaction of a single capillary bridge with its height varying from 6 to $25 \mathrm{~nm}$ and found that the shear interaction can be divided into elastic and damping forces due to contact angle hysteresis [23]. With atomic force microscopy or surface force apparatus (SFA), the friction of a capillary bridge with a diameter in micrometers has been experimentally studied and showed that the friction varies continuously with the height of the bridge as expected by macroscopic/continuum theory [24, 25]. There is little study on the friction caused by a capillary bridge with a height comparable to the size of single atom and a diameter in nanometers. Such a capillary bridge will be present when two smooth hydrophilic surfaces [26] come into contact. Another case where capillary bridges could play key roles is the capillary channel, such as a carbon nanotube filled with discrete liquid columns [27].

We systematically studied the effect of the capillary bridge on friction at the nanoscale. Using molecular dynamics (MD) simulation, we found that for a capillary bridge with a small radius confined between two hydrophilic elastic solid surfaces, the friction oscillates dramatically when decreasing the bridge height. The underlying mechanism was revealed to be a periodic ordered-disordered transition at the liquid-solid interfaces caused by the balance between the capillary force of the bridge and the bending stiffness of the surface. A parameter-free model was proposed based on such understanding and found to be in good agreement with the MD simulation results.

\section{Methods}

As shown in Figs. 1(a) and 1(b), the system is composed of two smooth solid surfaces with a nonvolatile capillary bridge confined between them. The surfaces are created by separating the (001) crystal planes of a face-centered-cubic (FCC) crystal cuboid where [ $\left.\begin{array}{lll}1 & 0 & 0\end{array}\right],\left[\begin{array}{lll}0 & 1 & 0\end{array}\right]$, and [ [ $\left.\begin{array}{lll}0 & 1 & 1\end{array}\right]$ direction is along the $x, y$, and $z$ axis, respectively. Each surface is composed of two layers of atoms. For a typical simulated system, there are 400 atoms for the capillary bridge and 3,200 atoms for the solid surfaces, i.e., 800 atoms within each solid layer. The mass of an individual atom in the solid surface is set to that of carbon atom. Since there are 1,600 atoms per plate, the mass per plate is $3.2 \times 10^{-23} \mathrm{~kg}$.

The bottom layer of the lower surface is fixed to the stationary stage. The top plate (slider) is coupled to a driven tip through three independent springs connecting the center of mass of the top plate with the tip. The force exerted on the top plate by the springs is evenly distributed among all the atoms within the top plate. The corresponding spring constants are $k_{x}=k_{y}=7 \mathrm{~N} / \mathrm{m}$ and $k_{z}=28 \mathrm{~N} / \mathrm{m}$, which are chosen to stand for the weak mechanical interactions with the substrate $[26,28]$. To avoid vibration of the upper plate, viscous damping $\eta$ is applied to the three springs with values corresponding to critical damping. The springs together with the damping provide reasonable constraints for the dynamics of the plate. A periodic boundary condition is applied in both the $x$ and $y$ direction. A vacuum along the $z$ direction is used to prevent interaction between the system and its images.

The interaction between atoms in the surfaces is described by a harmonic potential $U_{\text {har }}=k(r-d)^{2} / 2$, where $k$ is the spring constant and $r$ is the distance between neighboring atoms. The equilibrium bond distance $d=a / \sqrt{2}$ with $a$ being the lattice constant of the conventional unit cell of the FCC structure. The bond springs are connected between the atoms 
and their nearest neighbors. For the liquid atoms, we first describe their interactions with the LennardJones (LJ) potential as $U_{\mathrm{LL}}=4 \varepsilon\left[(\sigma / r)^{12}-(\sigma / r)^{6}\right]$, where $\varepsilon$ is the depth of the potential well and $\sigma$ is the distance at which the inter-particle potential is zero. In addition, to eliminate evaporation, we further combine every four liquid atoms into a short molecule chain. Such a constraint is achieved by applying a finitely extensible nonlinear elastic (FENE) potential $U_{\mathrm{FENE}}=-1 / 2 K R_{0}^{2} \ln \left[1-\left(r / R_{0}\right)^{2}\right]$ on the neighboring atoms within one single chain [8], where $K$ is the bead spring coefficient and $R_{0}$ is the maximum extent of the bond. As verified by Cheng and Robbins [9], the vapor pressure of the liquid in this case can be neglected without significantly increasing the molecular size or slowing down the dynamics. Aiming at capturing the generic behavior of the system, the mass of the liquid atoms is chosen to be the same as that of the solid. The interaction between the solid and liquid is described by the LJ potential with $U_{\mathrm{SL}}=4 \varepsilon_{\mathrm{SL}}\left[\left(\sigma_{\mathrm{SL}} / r\right)^{12}-\left(\sigma_{\mathrm{SL}} / r\right)^{6}\right]$, where $\varepsilon_{\mathrm{SL}}=0.8 \varepsilon$ and $\sigma_{\mathrm{SL}}=1.2 \sigma$. For both $U_{\mathrm{LL}}$ and $U_{\mathrm{SL}}$, the LJ potential is truncated and shifted at a cutoff radius $r_{\mathrm{c}}=2.5 \sigma$.

As we are interested in the properties of the system at steady state, we first equilibrate the system at $T$ for 20 million timesteps with the height of the tip remaining fixed. During this process, the distance between the two surfaces, $h$, is calculated as the vertical distance between the center of mass of the two inner layers (labeled red in Fig. 1). Then the tip was moved at a constant velocity, $v_{\mathrm{dr}}$, along the $+y$ direction with the height of the tip remaining fixed. When the temperature and momentum of the capillary bridge were stable, another 100 million timesteps were run for statistics. All simulations were performed using the large-scale atomic-molecular massively parallel simulator (LAMMPS) developed at Sandia National Laboratories [29].

In our simulations, we used dimensionless quantities with their units described in terms of $\varepsilon$, $\sigma$, and $m$. With $\varepsilon=43.7 \mathrm{meV}, \sigma=0.5 \mathrm{~nm}$, and $m=1 \times 10^{-25} \mathrm{~kg}$, the typical values of the quantities used are $a=2^{-1 / 3} \sigma, v_{\mathrm{dr}}=0.04 \sigma / \tau \approx 10 \mathrm{~m} / \mathrm{s}$ where $\tau=\sqrt{m \sigma^{2} / \varepsilon} \approx 1.88$ ps, $k=228 \varepsilon / \sigma^{2}, \quad K=30 \varepsilon / \sigma^{2}$, $R_{0}=1.5 \sigma$, and $T=350 \mathrm{~K}$. The corresponding time step of the velocity Verlet algorithm is $0.002 \tau$ which is about $4 \mathrm{fs}$.

\section{Results and discussion}

Figure 1(c) shows the lateral force, $F_{\mathrm{L}}$, i.e., friction, and normal force $F_{\mathrm{N}}$ exerted on the sliding surface by the capillary bridge as a function of the distance between surfaces, $h$. The volume of the nonvolatile confined liquid is kept constant for systems with different $h$. Both $F_{\mathrm{L}}$ and $F_{\mathrm{N}}$ show damped oscillations as $h$ increases and are out of phase with each other. For $F_{\mathrm{N}}$, its periodicity is due to molecular layering of the liquid confined in the narrow gap between the tip and substrate [8]. For $F_{\mathrm{L}}$, we note that similar behavior has been observed for two surfaces with

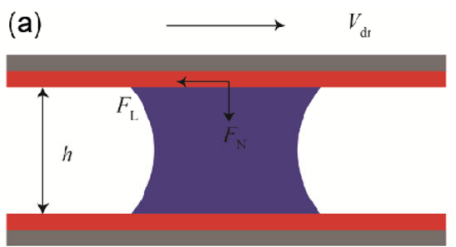

(b)

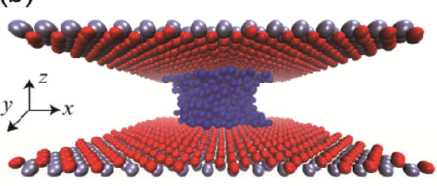

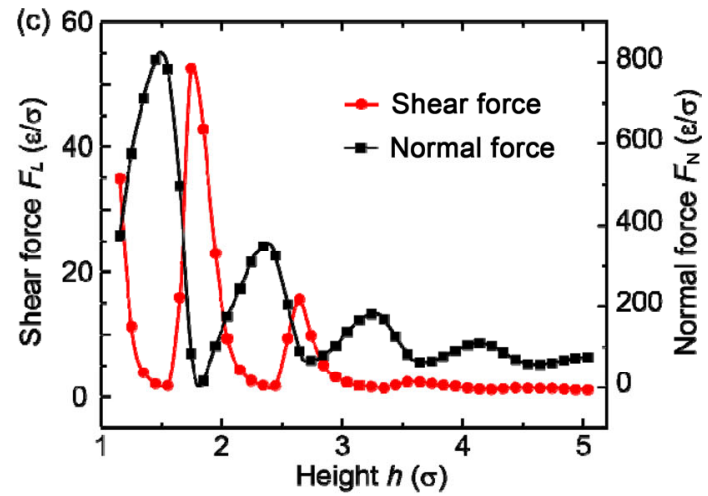

Fig. 1 (a) A schematic of the simulation system; the liquid is embedded between two solid plates. The upper plate is pulled by a spring with the other moving at constant speed, and the lower plate is fixed. (b) Each plate is composed of two layers of atoms. The outer layers (grey) were frozen and the inner layers (red) were equilibrated at room temperature. (c) The magnitude of shear and normal force experienced by the plate as a function of the distance between plates. 
the inter-surface space filled with liquid [30]. In this case, there is no capillary bridge and the oscillation is attributed to the ratio between the lattice size of the solid surface and the diameter of the liquid molecules [30].

To verify whether the mechanism as revealed in Fig. 1(c) also holds for our observations, we first calculated the number density of the liquid. As shown in Fig. 2(a), a layered structure is observed within the liquid. As $h$ decreases from 2.69 to 1.77, the number of layers gradually decreases from 4 to 3 , indicating a complete structural transition period happens in between. We calculated the structure factor for the layer of liquid next to the solid surface $S(q)$. The structure factor is calculated as $S(\boldsymbol{q})=\frac{1}{N} \sum_{j=1}^{N} \sum_{k=1}^{N} \mathrm{e}^{i \boldsymbol{q}\left(\boldsymbol{r}_{j}-\boldsymbol{r}_{k}\right)}$, where $\boldsymbol{r}_{j}$ and $\boldsymbol{r}_{k}$ represent the position of the $j^{\text {th }}$ and $k^{\text {th }}$ atom, $N$ is the amount of liquid atoms within the first layer, $q$ is the Hash lattice vector of the solid, and $<>$ represents the average value for the system in equilibrium [31, 32].
Clearly, within this layer of liquid, the structure changes periodically as $h$ decreases (Figs. 2(b)-2(d)), showing that this is the reason for the oscillating behavior in $F_{\mathrm{L}}$. The atomic structure corresponding to this transition is shown in Fig. S1 in the Electronic Supplementary Material (ESM). At the comensurate state, the capillary bridge experiences large friction, showing a stick-slip phenomenon, whereas at the disordered state, the force trace becomes erratic (Figs. 2(e) and 2(f)). The transition between ordered and disordered states accounts for the periodic changes in $F_{\mathrm{L}}$. Also, as $h$ decreases, the contact area between the capillary bridge and the solid surface increases, resulting in an inversely proportional relationship between $F_{\mathrm{L}}$ and $h$. Together with the ordered-disordered transition, these two mechanisms lead to the damped oscillating dependence of $F_{\mathrm{L}}$ on $h$ as shown in Fig. 1(c). In the systems studied here, due to the ultra-smooth surface, the friction originates solely within the contact area between the liquid and solid. This indicates a different
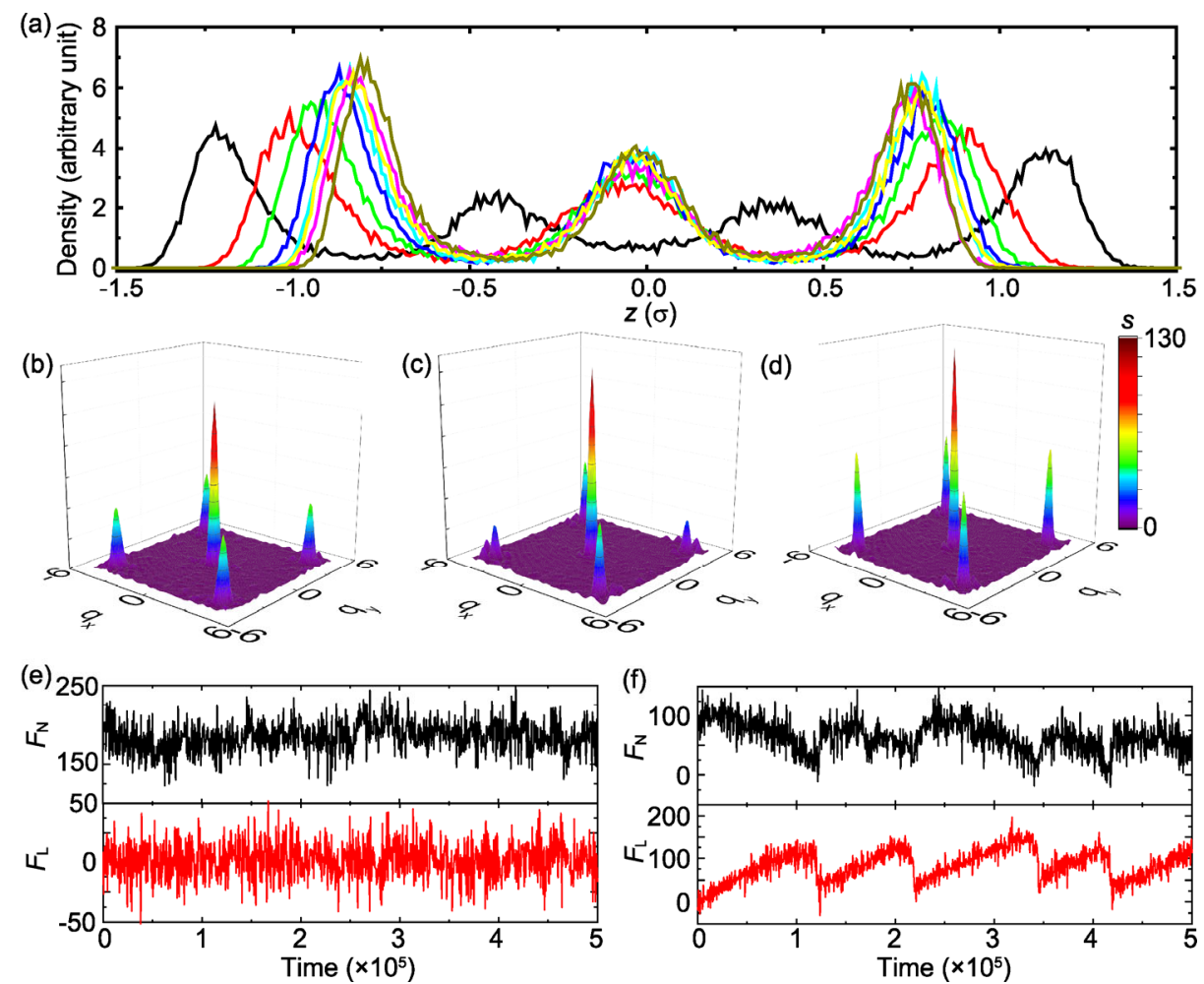

Fig. 2 (a) In a typical periodic phase for the height varying from 2.69 to $1.77(h=2.69,2.45,2.31,2.18,2.09,2.00,1.88,1.77)$, the number of liquid layers changes from 4 to 3; this number can be easily obtained by counting the peaks. Correspondingly, the structure factor between the solid and the neighboring liquid layer is shown in $(b-d)$, which indicates an ordered- (b for 4 layers, $\mathrm{d}$ for 3 layers) disordered (c for 3 layers) transition. (e, f) Lateral force and normal force for $h=2.45$ and 1.77 , respectively. 
mechanism from the stick-slip friction phenomenon observed when sliding an etched gold tip across a mica surface, which is caused by the three-phase contact line [23].

Compared with a slit filled with liquid, one distinct feature of the capillary bridge is its lateral size characterized by the contact radius $r$, i.e., the radius of its contact area with the solid surface. To investigate the dependence of $F_{\mathrm{L}}$ and $F_{\mathrm{N}}$ on $r$, we increase $r$ with $h$ being fixed. In this case, the volume of the capillary bridge is increased. Interestingly, we find that there is a critical $r_{\mathrm{c}}$ above which the dependence of $F_{\mathrm{L}}$ on $h$ becomes continuous (Fig. 3) while for $F_{\mathrm{N}}$ the discontinuity still holds. The remaining of discontinuity in $F_{\mathrm{N}}$ is easy to understand as it is decided by the instability of the system when $h$ increases. For liquid confined between surfaces, obviously this critical behavior is unique to the capillary bridge, and to our best knowledge, this critical radius phenomenon has never been reported.

The key to understanding the existence of the critical radius is the capillary force present near the contact line [33] along with the Laplace pressure, which is unique to capillary bridges. The capillary force induces a normal force on the surface, $F_{\gamma}=2 \pi \gamma r \sin \theta$, where $\gamma$ is the surface tension of the liquid-vapor interface and $\theta$ is the contact angle. The Laplace pressure also exerts a normal force on the surfaces, $F_{\Delta P}=\pi r^{2} \Delta P$, where $\Delta P$ is the pressure drop across the surface of the capillary bridge. From the Young-Laplace theory, the pressure drop can be estimated as $\Delta P=2 \gamma \kappa=\gamma\left(1 / r_{1}+1 / r_{2}\right)$, where $r_{1}$ and $r_{2}$ are the principle curvatures of the surface of the capillary bridge. Thus, the force generated by the Laplace pressure is $F_{\Delta P}=\pi r^{2} \gamma\left(1 / r_{1}+1 / r_{2}\right)=$ $\pi \gamma r\left(r / r_{1}+r / r_{2}\right)$. For hydrophilic surfaces as studied here, it is trivial to show that $r_{1}=r-h / 2 \cdot(1-$ $\tan \theta / 2) /(1+\tan \theta / 2)$ and $r_{2}=h / 2 \cos \theta$. By noting that the critical radius $r_{\mathrm{c}}$ is much larger than the distance between surfaces $h$, i.e., $r \gg h$ when $r=r_{\mathrm{c}}$, $r_{2} \leqslant h / 2 \ll r$, we have $r / r_{1}+r / r_{2} \gg 2$. In this case $F_{\Delta P} \gg 2 \pi \gamma r \geqslant F_{\gamma}$. Given that the total force exerted on the surfaces by the capillary bridge $F_{\text {cap }}=F_{\gamma}+F_{\Delta P}$ and $F_{\Delta P} \gg F_{\gamma}, F_{\text {cap }}$ can be approximated as

$$
F_{\text {cap }} \approx F_{\Delta P}=\pi \gamma r\left(r / r_{1}+r / r_{2}\right) \approx 2 \cos \theta \pi \gamma r^{2} / h=\pi r^{2} \Delta P
$$

by noting that $r_{1} \approx r$. The approximated pressure drop $\Delta P=2 \gamma \cos \theta / h$ used in Eq. (1) has been adopted by de Gennes [7].

Evidently, the force $F_{\text {cap }}$ induces deformation of the surfaces as shown in Fig. 4(a) and illustrated in Section 2 of the ESM. According to the KirchhoffLove plate theory $\nabla^{2} \nabla^{2} w=\Delta P / D$, where $w$ is the deflection of the surface and $D$ is the bending stiffness. Bending stiffness is determined by $D=E H^{3} / 12\left(1-v^{2}\right)$, where $E$ is the elastic modulus, $H$ is the thickness of the plate, and $v$ is Poisson ratio. According to the solution given by Poisson in 1829 [34], $w=\Delta P\left(x^{2}-r^{2}\right)^{2} / 64 D$, where $x$ is the distance to the center of the contact area. When $x=0$, we get the maximum deflection $w_{\mathrm{m}}=3(1-$ $\left.v^{2}\right) \gamma \cos \theta r^{4} / 8 E H^{3} h$.

When $w_{\mathrm{m}}$ is large enough, within the contact area between the solid surface and the liquid, different regions experience different lateral forces as shown in Fig. 4(b); the overall resistance exerted on the sliding surface will be the average of all the lateral forces. For example, in our case when $w_{\mathrm{m}} \geqslant$ $\delta_{0} \approx \delta / 5$, all states corresponding to different lateral
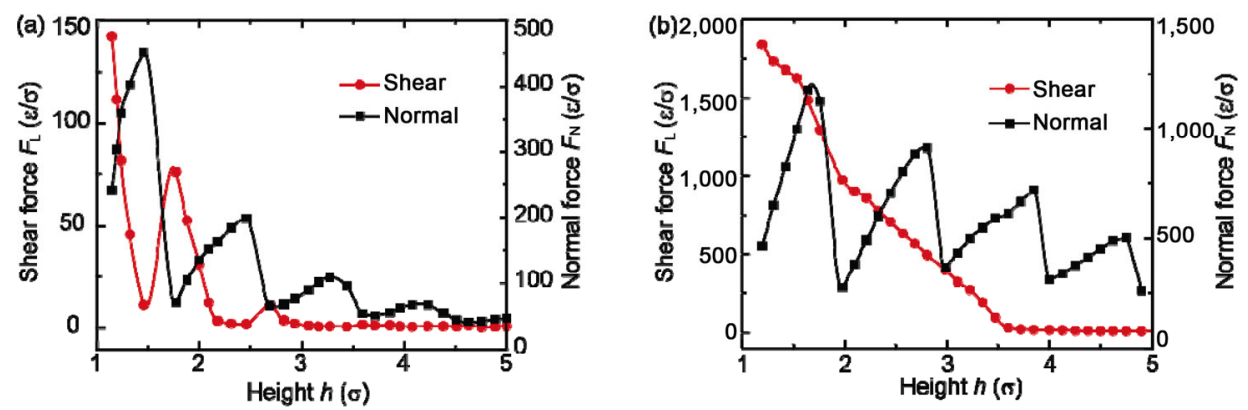

Fig. 3 Comparison of $F_{\mathrm{L}}$ and $F_{\mathrm{N}}$ for a capillary bridge with (a) $r=9.52$ and (b) $r=11.50$ when $h$ is 5.0. As $r$ increases, the oscillating dependence of $F_{\mathrm{L}}$ on $h$ disappears, but remains for $F_{\mathrm{N}}$. 
(a)

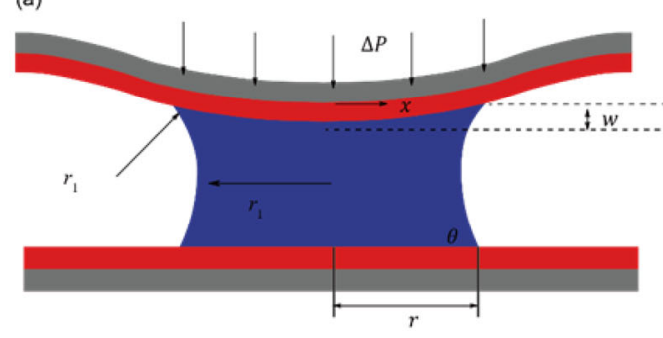

(b)

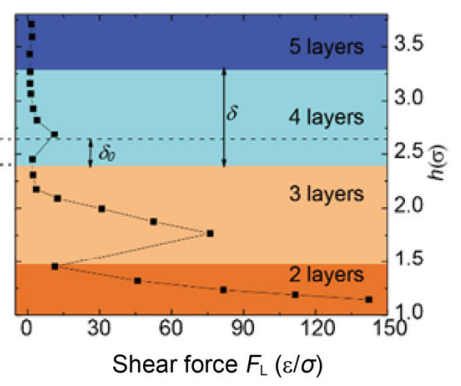

Fig. 4 (a) Schematic of the deformation of the plate and the capillary bridge under the pressure differential caused by surface tension. The magnitude of the deformation is enlarged for better visibility. (b) The corresponding shear force as a function of height.

forces are covered (corresponding to points with $2.43<h<2.76$ in Fig. 4(b)). In this case, the discontinuity in $F_{\mathrm{L}}$ is eliminated. Thus, the critical condition for the elimination of the discontinuity in $F_{\mathrm{L}}$ is $3\left(1-v^{2}\right) \gamma \cos \theta r^{4} / 8 E H^{3} h=w_{0}$, where $w_{0}$ is the minimum range within which the lateral force reaches its maximum and minimum as $h$ changes for a capillary bridge with a small radius and height (in our case $w_{0}=\delta / 5$ ). Thus, the critical radius predicted theoretically, $r_{c}$, can be estimated as

$$
r_{\mathrm{c}}=\left(8 E H^{3} h w_{0} / 3\left(1-v^{2}\right) \gamma \cos \theta\right)^{1 / 4}
$$

In the system studied, $E=240, v=0.33, H=1.62$, $\delta=0.78, \gamma=0.88, \theta=75^{\circ}, h=5.0$, and $w_{0}=\delta / 5$; the critical radius predicted theoretically is $r_{\mathrm{c}}=10.12$. This is in excellent agreement with the results from the direct MD simulations which were $9.52<$ $r_{\mathrm{c}}<11.50$. It should be noted that the transition only holds when $F_{\mathrm{L}}$ shows a discontinuous dependence on $h$ for a capillary bridge with small radius, i.e., $h$ is small (comparable to $\sigma$ ).

As shown in Eq. (2), our model predicts that the critical condition is also determined by the elastic modulus and the contact angle. In the MD simulation, the elastic modulus can be adjusted by changing the harmonic potential parameter $K$, and the contact angle can be adjusted by changing the depth of the potential well of the LJ potential $\varepsilon_{\mathrm{LS}}$ between the solid and liquid. By changing $E$ and $\theta$ within the direct MD simulations, we plotted the phase diagram for the transition in $F_{\mathrm{L}}$ as a function of $h$ (Fig. 5). The critical transition estimated from the MD simulations are in excellent agreement with the prediction by our analytic model (Eq. (2)).

In experiments with $h$ on the order of nanometers,

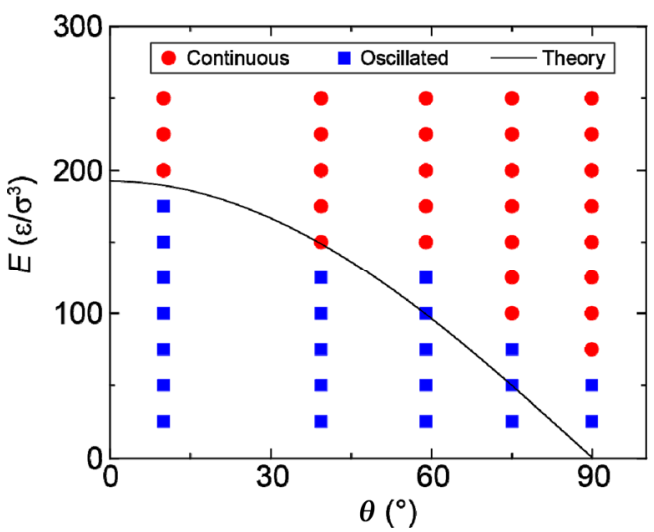

Fig. 5 Phase diagram of the shear modes characterized by contact angle and elastic modulus. The stepped mode is in red and the continuous mode is in blue. The critical transition as predicted by Eq. (2) is plotted as a solid curve.

the radius of capillary bridge is usually larger than the critical radius, which makes it hard to observe the oscillation in the lateral force. However, with our understanding as quantitatively summarized in Eq. (2), it is possible to observe the oscillation by controlling the radius of the capillary bridge, the distance between the surfaces, the elastic modulus of the surface, and by using a certain liquid. As a rule of thumb for experimentalists, in Table 1, we list the critical radius, $r_{\mathrm{c}}$, for some typical friction pairs with confined liquid at the interface [35]. A potential experimental system could be graphene layers deposited by exfoliation onto a smooth surface (e.g., a $\mathrm{Si} / \mathrm{SiO}_{2}$ surface), which represent a large category of experimental systems [26, 36, 37]. For such systems, it has been shown experimentally that the few-layer graphene is partly freely suspended between hills of the substrate [26]. Thus, when two hydrophilic surfaces [38] of this kind slide against 
Table 1 Critical radius $r_{\mathrm{c}}$ for some typical capillary bridge systems, $H$ is about $1 \mathrm{~nm}$ in each condition, $E^{*}=E /\left(1-v^{2}\right)$, the effective elastic modulus.

\begin{tabular}{lccccccc}
\hline Solid & Liquid & $\begin{array}{c}E^{*} \\
(\mathrm{GPa})\end{array}$ & $\begin{array}{c}\theta \\
\left({ }^{\circ}\right)\end{array}$ & $\begin{array}{c}\delta \\
(\mathrm{nm})\end{array}$ & $\begin{array}{c}\gamma \\
(\mathrm{nN} / \mathrm{nm})\end{array}$ & $\begin{array}{c}h \\
(\mathrm{~nm})\end{array}$ \\
\hline Graphene & Water & $\sim 2,000$ & 85 & 0.3 & 0.073 & $0.3-1.6$ & $11.1-16.8$ \\
Silicon & Water & $\sim 200$ & 40 & 0.3 & 0.073 & $0.3-1.6$ & $3.6-5.5$ \\
Gold & Water & $\sim 100$ & 60 & 0.3 & 0.073 & $0.3-1.6$ & $3.4-5.1$ \\
Glass & {$[\mathrm{C} 4 \mathrm{C} 1$ im] $]$ NTf2] } & 10 & 25 & $\sim 1$ & 0.04 & $0.1-0.5$ & $2.0-3.0$ \\
Silicon & {$[\mathrm{C} 4 \mathrm{C} 1$ im] $][$ NTf2] } & $\sim 200$ & 35 & $\sim 1$ & 0.04 & $0.1-0.5$ & $4.2-6.3$ \\
\hline
\end{tabular}

each other under ambient conditions, their frictional process may be well described by our model.

\section{Conclusions}

In recent years, there have been some studies about the properties of liquid bridges. However, there are very limited studies on the friction of capillary bridges at the nanoscale [23-25, 27, 39]. For a capillary bridge between hydrophilic surfaces, we observed an oscillating dependence of friction on the distance between surfaces using MD simulations. The behavior of the normal force in our simulation coincided with the findings of Cheng and Robbins [9]. Moreover, with a large scale simulation, we verified that the friction varies continuously with the height of the bridge as expected by macroscopic continuum theory $[24,25]$. Interestingly, there is a critical size below which the friction oscillates. Usually, macroscopic capillary theory [40] and elasticity theory [41] can be extended to the nanoscale. However, we found an entirely different change of friction at the nanoscale compared to the macroscale.

The underlying mechanism was revealed to be the balance between the capillary force of the capillary bridge and the bending stiffness of the surface. The parameter-free analytical model proposed agrees well with the simulation results, thus providing a guideline for controlling friction between hydrophilic surfaces at distances on the order of nanometers, e.g., dip-pen nanolithography where smooth kinetics is required for the tip movement [42].

\section{Acknowledgements}

M.M. wishes to acknowledge the financial support by Thousand Young Talents Program and the National
Natural Science Foundation of China (Nos. 11632009 and 11772168).

Electronic Supplementary Material: Suppleenary material is available in the online version of this article at https://doi.org/10.1007/s40544-020-0396-x.

Open Access: This article is licensed under a Creative Commons Attribution 4.0 International License, which permits use, sharing, adaptation, distribution and reproduction in any medium or format, as long as you give appropriate credit to the original author s) and the source, provide a link to the Creative Commons licence, and indicate if changes were made.

The images or other third party material in this article are included in the article's Creative Commons licence, unless indicated otherwise in a credit line to the material. If material is not included in the article's Creative Commons licence and your intended use is not permitted by statutory regulation or exceeds the permitted use, you will need to obtain permission directly from the copyright holder.

To view a copy of this licence, visit http://creativecommons.org/licenses/by/4.0/.

\section{References}

[1] Xiao X, Qian L. Investigation of humidity-dependent capillary force. Langmuir 16: 8153-8158 (2000)

[2] Choe H, Hong M H, Seo Y, Lee K, Kim G, Cho Y, Ihm J, Jhe W. Formation, manipulation, and elasticity measurement of a nanometric column of water molecules. Phys Rev Lett 95: 187801 (2005)

[3] Bocquet L, Charlaix E, Ciliberto S, Crassous J. Moisturenduced ageing in granular media and the kinetics of capillary condensation. Nature 396: 735 (1998)

[4] Restagno F, Bocquet L, Biben T. Metastability and 
nucleation in capillary condensation. Phys Rev Lett $\mathbf{8 4}$ : 2433-2436 (2000)

[5] Jang J, Schatz G C, Ratner M A. How narrow can a meniscus be? Phys Rev Lett 92: 085504 (2004)

[6] Dörmann M, Schmid H-J. Simulation of capillary bridges between nanoscale particles. Langmuir 30: 1055-1062 (2014)

[7] Gennes P G D, Brochard-Wyart F, Quéré D. Capillarity and Wetting Phenomena. Springer, 2004.

[8] Cheng S, Robbins M O. Capillary adhesion at the nanometer scale. Phys Rev E 89: 062402 (2014)

[9] Cheng S, Robbins M O. Nanocapillary adhesion between parallel plates. Langmuir 32: 7788 (2016)

[10] Valenzuela G E, Rozas R E, Toledo P G. Molecular dynamics simulation of nanoforces between substrates mediated by liquid bridges: Controlling separation and force fluctuations. J Phys Chem C 121: 25986-25993 (2017)

[11] Riedo E, Lévy F, Brune H. Kinetics of capillary condensation in nanoscopic sliding friction. Phys Rev Lett 88: 185505 (2002)

[12] Lancaster J K. A review of the influence of environmental humidity and water on friction, lubrication and wear. Tribol Int 23: 371-389 (1990)

[13] Popov V L. Contact Mechanics and Friction: Physical Principles and Applications, 2nd Ed. Springer, 2017.

[14] Raviv U, Laurat P, Klein J. Fluidity of water confined to subnanometre films. Nature 413: 51-54 (2001)

[15] Zangi R, Mark A E. Monolayer ice. Phys Rev Lett 91: 025502 (2003)

[16] Jinesh K B, Frenken J W M. Experimental evidence for ice formation at room temperature. Phys Rev Lett 101: 036101 (2008)

[17] Cao W, Wang J, Ma M. Mechano-nanofluidics: Water transport through CNTs by mechanical actuation. Microfluid Nanofluid 22: 125 (2018)

[18] Bo N J P, Spencer N D. Sliding friction: Physical principles and applications. Phys Today 52: 66-68 (1999)

[19] Szoszkiewicz R, Riedo E. Nucleation time of nanoscale water bridges. Phys Rev Lett 95: 135502 (2005)

[20] Noel O, Mazeran P-E, Nasrallah H. Sliding velocity dependence of adhesion in a nanometer-sized contact. Phys Rev Lett 108: 015503 (2012)

[21] Barel I, Filippov A E, Urbakh M. Formation and rupture of capillary bridges in atomic scale friction. $J$ Chem Phys 137: 164706 (2012)

[22] Capozza R, Barel I, Urbakh M. Effect of Capillary Condensation on Nanoscale Friction. Springer International Publishing, 2015.

[23] Lee M, Kim B, Kim J, Jhe W. Noncontact friction via capillary shear interaction at nanoscale, Nat Commun 6: 7359 (2015)
[24] Becker T, Mugele F. Nanofluidics: Viscous dissipation in layered liquid films. Phys Rev Lett 91: 166104 (2003)

[25] Bureau L. Nonlinear rheology of a nanoconfined simple fluid. Phys Rev Lett 104: 218302 (2010)

[26] Geringer V, Liebmann M, Echtermeyer T, Runte S, Schmidt M, Ruckamp R, Lemme M C, Morgenstern M. Intrinsic and extrinsic corrugation of monolayer graphene deposited on $\mathrm{SiO}_{2}$. Phys Rev Lett 102: 076102 (2009)

[27] Tunuguntla R H, Henley R Y, Yao Y C, Pham T A, Wanunu M, Noy A. Enhanced water permeability and tunable ion selectivity in subnanometer carbon nanotube porins. Science 357: 792 (2017)

[28] Ouyang W, Ma M, Zheng Q, Urbakh M. Frictional properties of nanojunctions including atomically thin sheets. Nano Lett 16: 1878-1883 (2016)

[29] Plimpton S. Fast parallel algorithms for short-range molecular dynamics. J Comput Phys 117: 1-19 (1995)

[30] Neek-Amal M, Peeters F M, Grigorieva I V, Geim A K. Commensurability effects in viscosity of nanoconfined water. ACS Nano 10: 3685-3692 (2016)

[31] Falk K, Sedlmeier F, Joly L, Netz R R, Bocquet L. Molecular origin of fast water transport in carbon nanotube membranes: Superlubricity versus curvature dependent friction. Nano Lett 10: 4067 (2010)

[32] Allen M P, Tildesley D J. Computer Simulation of Liquids. New York (US): Oxford University Press, 1987.

[33] Wu S, Ma M. A contact angle hysteresis model based on the fractal structure of contact line. $J$ Colloid Interface Sci 505: 995-1000 (2017)

[34] Timoshenko S P, Woinowsky-Krieger S. Theory of Plates and Shells. McGraw-hill, 1959.

[35] Ge X, Halmans T, Li J, Luo J. Molecular behaviors in thin film lubrication-Part three: Superlubricity attained by polar and nonpolar molecules. Friction 7: 625-636 (2019)

[36] Liu L, Zhou M, Jin L, Li L, Mo Y, Su G, Li X, Zhu H, Tian Y. Recent advances in friction and lubrication of graphene and other 2D materials: Mechanisms and applications. Friction 7: 199-216 (2019)

[37] Gongyang Y, Ouyang W, Qu C, Urbakh M, Quan B, Ma M, Zheng Q. Temperature and velocity dependent friction of a microscale graphite-DLC heterostructure. Friction 8: 462-470 (2020)

[38] Ma M, Tocci G, Michaelides A, Aeppli G. Fast diffusion of water nanodroplets on graphene. Nature Mater 15: 66-71 (2016)

[39] Sung B, Kim J, Stambaugh C, Chang S J. Direct measurement of activation time and nucleation rate in capillary-condensed water nanomeniscus. Appl Phys Lett 103: 236102 (2013) 
[40] Giovambattista N, Almeida A B, Alencar A M, Buldyrev $\mathrm{S}$ V. Validation of capillarity theory at the nanometer scale by atomistic computer simulations of water droplets and bridges in contact with hydrophobic and hydrophilic surfaces. J Phys Chem C 120: 1597-1608 (2016)

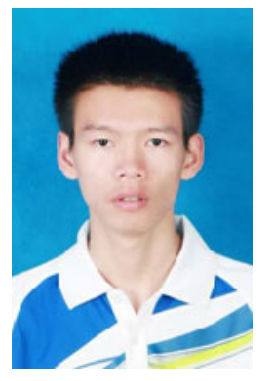

Shuai WU. He received his bachelor degree in civil engineering in 2012 from Nanchang University, Nanchang, China. Then, he studied for his doctorate in the Department

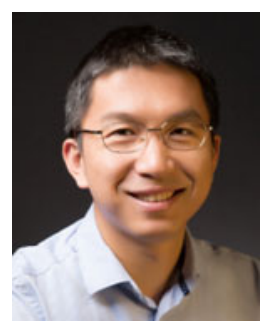

Ming MA. He received his Ph.D. degree in engineering mechanics from Tsinghua University, China, in 2011. He joined the State Key Laboratory of Tribology at Tsinghua
[41] Duan H, Wang J, Karihaloo B L. Theory of elasticity at the nanoscale. Adv Appl Mech 42: 1-68 (2009)

[42] Piner R D, Zhu J, Xu F, Hong S, Mirkin C A. "Dip-pen" nanolithography. Science 283: 661-663 (1999)

of Engineering Mechanics at Tsinghua University. His research interests include nano/micro mechanics and super hydrophobicity.

University since 2016. His current position is an associate professor. His research areas cover nanotribology, nanofluidics, superlubricity, and diffusion on surfaces or under confinement. 